\title{
Subthalamic Local Field Beta Oscillations during Ongoing Deep Brain Stimulation in Parkinson's Disease in Hyperacute and Chronic Phases
}

\author{
Manuela Rosa ${ }^{a}$ Gaia Giannicola ${ }^{a}$ Domenico Servello ${ }^{b}$ Sara Marceglia ${ }^{a}$ \\ Claudio Pacchettic Mauro Porta ${ }^{b}$ Marco Sassi ${ }^{b}$ Emma Scelzo $^{a}$ \\ Sergio Barbieri ${ }^{d} \quad$ Alberto Priori $^{\mathrm{a}}{ }^{\mathrm{e}}$ \\ ${ }^{a}$ Centro Clinico per la Neurostimolazione, le Neurotecnologie ed i Disordini del Movimento, Fondazione IRCCS \\ $\mathrm{Ca}^{\prime}$ Granda, Ospedale Maggiore Policlinico, e b Neurochirurgia Funzionale e Clinica Tourette, IRCCS Galeazzi, \\ Milano, 'Unità Operativa Parkinson e Disordini del Movimento, IRCCS Istituto Neurologico Mondino, Pavia, \\ 'Unità Operativa di Neurofisiopatologia Clinica, Fondazione IRCCS Ca' Granda, Ospedale Maggiore Policlinico, e \\ e'Dipartimento di Scienze Neurologiche, Università degli Studi di Milano, Milano, Italia
}

\section{Key Words}

Subthalamic nucleus $\cdot$ Local field potential $\cdot$ Deep brain stimulation • Chronic recording $\cdot$ Hyperacute recording $\cdot$ Parkinson's disease $\cdot$ Beta activity $\cdot$ Adaptive deep brain stimulation system

\footnotetext{
Abstract

In the past years, local field potential (LFP) signals recorded from the subthalamic nucleus (STN) in patients undergoing deep brain stimulation (DBS) for Parkinson's disease (PD) disclosed that DBS has a controversial effect on STN beta oscillations recorded 2-7 days after surgery for macroelectrode implantation. Nothing is known about these DBS-induced oscillatory changes 30 days after surgery. We recorded STN LFPs during ongoing DBS in 7 patients with PD, immediately (hyperacute phase) and 30 days (chronic phase) after surgery. STN LFP recordings showed stationary intranuclear STN beta LFP activity in hyperacute and chronic phases, confirming that beta peaks were also present in chronic recordings. Power spectra of nuclei with significant beta activity ( $54 \%$ of the sample) showed that it decreased significantly during DBS $(p=0.021)$ under both recording conditions. The
}

time course of beta activity showed more evident DBS-induced changes in the chronic than in the hyperacute phase $(p=0.014)$. DBS-induced changes in STN beta LFPs in patients undergoing DBS in chronic phase provide useful information for developing a new neurosignal-controlled adaptive DBS system.

Copyright $\odot 2011$ S. Karger AG, Base

\section{Introduction}

Neuronal activity around the macroelectrodes implanted in the human subthalamic nucleus (STN) for deep brain stimulation (DBS) produces oscillations known as local field potentials (LFPs) [1-3]. These signals provided physiological information about STN responses to various stimuli [4-9].

Over recent years, studies describing STN LFP signals recorded during DBS have done much to explain the mechanisms underlying DBS in patients with Parkinson's disease (PD) [10-16]. Studies investigating DBS-induced changes in STN LFPs focused on rhy thms oscillating from the low-frequency band $(1-7 \mathrm{~Hz})$ to the beta

\section{KARGER}

Fax +4161306 1234

E-Mail karger@karger.ch

www.karger.com (c) 2011 S. Karger AG, Basel

$1424-862 X / 11 / 0193-0151 \$ 38.00 / 0$

Accessible online at:

www.karger.com/nsg
Alberto Priori, MD, PhD, Dipartimento di Scienze Neurologiche

Università degli Studi di Milano, Centro Clinico per la Neurostimolazione,

le Neurotecnologie ed i Disordini del Movimento, Fondazione IRCCS Ca' Granda

Ospedale Maggiore Policlinico, Via F. Sforza 35, IT-20122 Milano (Italy)

Tel.+39025503 3621, E-Mail alberto.priori@unimi.it 
Table 1. Patients' clinical details

\begin{tabular}{llllll}
\hline Patient & $\begin{array}{l}\text { Age } \\
\text { years }\end{array}$ & Gender & $\begin{array}{l}\text { Years of } \\
\text { disease }\end{array}$ & $\begin{array}{l}\text { Predominant } \\
\text { motor symptoms }\end{array}$ & $\begin{array}{l}\text { UPDRS III scores } \\
\text { pre-surgery } \\
\text { ON/OFF levodopa }\end{array}$ \\
\hline 1 & 71 & M & 5 & Rigidity & $30 / 44$ \\
2 & 70 & M & 7 & Tremor, dyskinesias & $19 / 42$ \\
3 & 69 & M & 10 & Tremor & $12 / 31$ \\
4 & 59 & M & 5 & Tremor & $18 / 35$ \\
5 & 58 & M & 13 & Tremor, freezing, dyskinesias & $18 / 34$ \\
6 & 72 & M & 10 & Rigidity, tremor & $21 / 40$ \\
7 & 69 & F & 5 & Tremor, rigidity, dyskinesias & $20 / 38$ \\
\hline
\end{tabular}

band $(8-35 \mathrm{~Hz})$. In particular, low-frequency STN oscillations increased during ongoing STN DBS [16] confirming previous observations that power in the $1-1.5 \mathrm{~Hz}$ band increased and slowly decayed to baseline levels after DBS was turned off [15].

Nevertheless, DBS-induced changes in STN beta oscillations remain controversial. Some investigators reported that after short-term and long-term STN DBS, beta oscillations decreased both in the STN itself and in the globus pallidus internus (GPi) [13, 17-20]. Conversely, our recordings soon after STN DBS [11] and during STN DBS [16, 21] failed to confirm the beta activity decrease. More recently, two studies reported that the beta oscillations significantly decreased during DBS, but did so only in parkinsonian patients whose LFP recordings already showed high-beta activity at baseline $[10,12]$. These studies documented changes in STN beta LFPs in PD patients only during the first week after DBS macroelectrode implantation.

A subsequent study then investigated the time-dependent STN LFP changes after DBS surgery in PD, and provided new evidence showing that DBS macroelectrode impedance and STN LFP patterns are a function of the time elapsing between macroelectrode implantation and LFP recording [22]. The time course of macroelectrode impedance is related to changes in the electrode/tissue interface and in the STN LFP low-frequency activity. Conversely, macroelectrode impedance is unrelated to beta activity, hence beta power recorded 30 days after surgery for macroelectrode positioning (chronic condition) is similar to power recorded 2-7 days thereafter [22].

None investigated how DBS changes the STN LFP beta activity in the longer term. Having this information would be useful for developing new adaptive DBS systems based on STN-LFP signal feedback designed to adapt DBS parameters to individual patient's clinical state.
In this paper, to clarify how DBS acts in the long term, we investigated changes induced by DBS in STN beta LFP in patients with PD 30 days after macroelectrode implantation. To do so, we recorded STN LFPs in 7 patients with PD during ongoing DBS immediately (hyperacute phase) and 30 days (chronic phase) after surgery.

\section{Patients and Methods}

\section{Patients}

Seven patients (6 men) with idiopathic PD and fulfilling the specific inclusion criteria for DBS treatment were studied after their informed consent and local institutional review board approval [23]. The study conformed with the Declaration of Helsin$\mathrm{ki}$. All the patients, except one, were bilaterally implanted in the STN with DBS macroelectrodes at the Functional Neurosurgery Unit of the Institute Galeazzi IRCCS in Milan, Italy. The other patient was studied during unilateral re-implantation of DBS macroelectrodes in the STN (patient 5 in table 1). Clinical details, including pre-surgery assessment of the Unified Parkinson's Disease Rating Scale III (UPDRS III, motor part), are reported in table 1.

\section{Surgical Procedures}

Surgical procedures are reported elsewhere [22]. In brief, the procedure for DBS consisted of a first surgery for macroelectrode placement in the STN followed by a second surgery to implant the subcutaneous pulse generator [24].

Between the two operations, macroelectrodes were not connected to the stimulator and were accessible for neurophysiological recordings. In our protocol, LFPs were first recorded from implanted macroelectrodes immediately at the end of the first surgery, when the patient was still in the operating room (hyperacute phase). Then, to enable LFP recordings during DBS in the longer term we postponed the pulse generator implant, 30 days after macroelectrodes positioning [22]. After the first operation, the implanted macroelectrodes were left under the skin so that the patient could be safely discharged. The stimulator was not implanted and the patient did not receive DBS during this time. About 30 days later, patients were again hospitalized and under- 
Table 2. Patients' electrode position and recording configuration

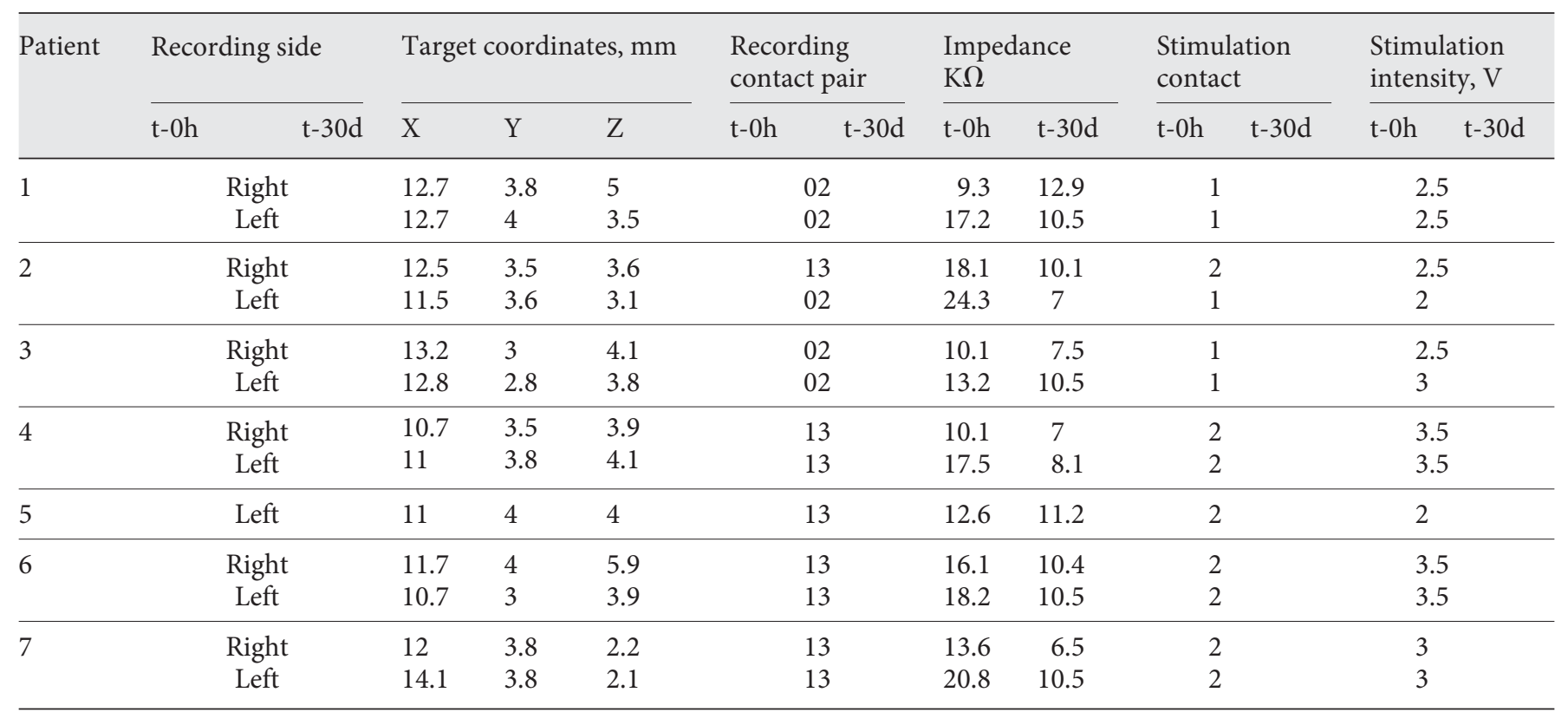

Target coordinates related to final position of contact 0 . Target coordinates were accorded to the anterior commissural-posterior commissural (AC-PC) line and midcommissural point (MCP). $\mathrm{X}=$ Lateral from midline; $\mathrm{Y}=$ posterior from $\mathrm{MCP}$; $\mathrm{Z}=$ ventral to ACPC line.

went surgery to implant the subcutaneous pulse generator. Before the operation, the macroelectrodes were externalized to allow LFP recordings during DBS 30 days after the first surgery (chronic phase).

The implanted 3389 Medtronic macroelectrode had four cylindrical contacts (diameter $1.27 \mathrm{~mm}$, length $1.5 \mathrm{~mm}$, placed $2 \mathrm{~mm}$ apart center-to-center) denominated $0-1-2-3$, beginning from the more caudal contact. The STN was targeted by visualization through a CT-MRI fusion-based technique before surgery [25]. The site of macroelectrode implantation was adjusted during surgery with micro-recordings and by clinically assessing changes induced by stimulation through the microelectrodes and through the implanted macroelectrodes. For all nuclei studied, postoperative MRI was obtained to assess the accuracy of final position of DBS macroelectrodes and to verify the placement of contact 0 within the STN [24] (table 2).

\section{Experimental Protocol and LFP Recordings}

LFP activity was recorded in two experimental sessions: hyperacute phase (t-0h) and chronic phase (t-30d) (fig. 1). All patients were studied at least $8 \mathrm{~h}$ after withdrawal from antiparkinsonian medication (off levodopa).

Each experimental session lasted approximately $30 \mathrm{~min}$ and took place in the operating room with the following steps: (1) evaluation of the impedance of each macroelectrodes' contact pair; (2) stimulation threshold: clinical assessment of optimal stimulation intensity followed by a pause for rest; (3) baseline: LFP recording at rest for about $2 \mathrm{~min}$; (4) DBS on: LFP recording during high- frequency stimulation for about 6 min, and (5) DBS off: LFP recording after high-frequency stimulation for about $2 \mathrm{~min}$.

Recording contact pairs and stimulation intensity were selected during the first recording session (hyperacute phase) and used also to record LFPs in the second session (chronic phase). Of the 7 participants, 6 underwent the recording procedures for the implanted macroelectrodes on both sides (right side first) and 1 patient for the re-implanted macroelectrode side (right side).

Macroelectrode impedance was evaluated through an impedance meter at $30 \mathrm{~Hz}$ (Model EZM 4; Grass, USA). Impedance was evaluated on all contact pairs $(0-1,0-2,0-3,1-2,1-3$ and $2-3)$. The contact pair ( $0-2$ or $1-3)$ showing the highest impedance value was selected for the bipolar recording, leaving the central contact (1 or 2) free for delivering DBS. LFPs were recorded through the FilterDBS system for artifact-free recording [21]. Two standard skin $\mathrm{Ag} / \mathrm{AgCl}$ electrodes (RedDot; 3M, USA) placed on the left and on the right supraclavicular area were used as recording and stimulation references respectively [16]. For electrical stimulation we used a constant voltage stimulator (Dual Screen; Medtronic, Minneapolis, Minn., USA) and DBS was delivered with a pulse width of $60 \mu \mathrm{s}$ and frequency of $130 \mathrm{~Hz}$ at a previously tested optimal stimulation intensity. Optimal stimulation intensity was set according to the stimulation threshold represented by the highest stimulation intensity that induced therapeutic effects without side effects. The threshold for each nucleus was established by a neurologist during the first recording session.

The recorded signals were amplified $(50,000 \times)$ and filtered $(0.5-45 \mathrm{~Hz})$ through the FilterDBS, then digitized through the 


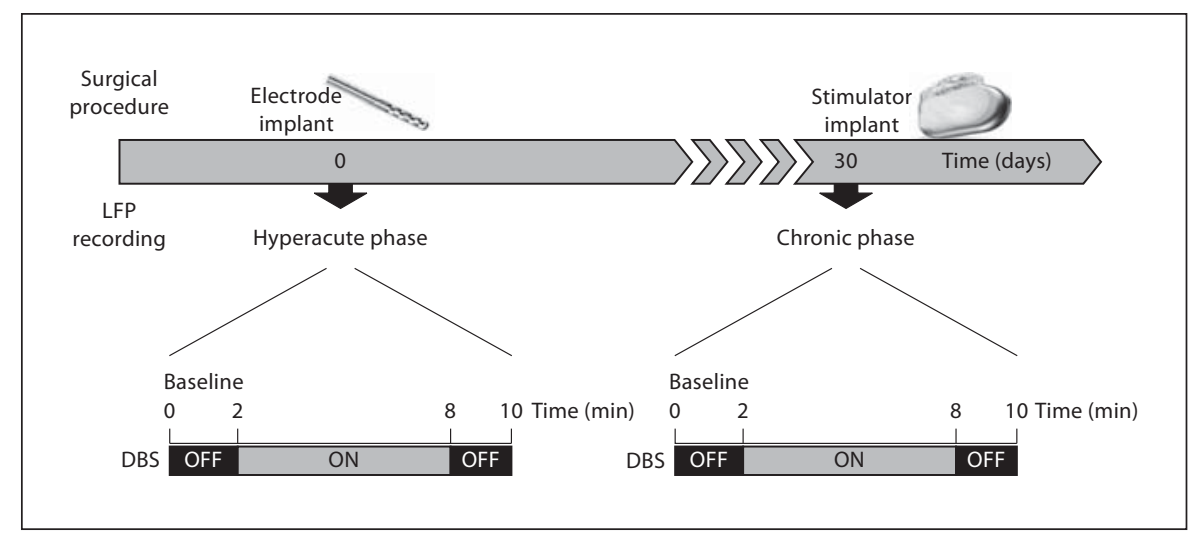

Fig. 1. Surgical procedure and experimental protocol. Timeline of deep brain stimulation (DBS) surgical procedure and local field potential (LFP) recordings for the experimental protocol. The pulse generator was placed and connected to macroelectrode 30 days after macroelectrode positioning. LFPs were recorded at two time points after macroelectrode implantation: the hyperacute

USB-6251 multifunctional device with 8 inputs (National Instruments Corp., Austin, Tex., USA) at 500 sample/s and 16-bit resolution with $10 \mathrm{~V}$ range. Recorded signals were displayed online on a PC monitor with LabVIEW Signal Express software (version 2.5; National Instruments Corp.) and stored for off-line analysis.

\section{Data Analysis}

Data were analyzed off-line with Matlab software (version 7.10; The MathWorks, Natick, Mass., USA). Signals were preliminarily band-passed $(2-45 \mathrm{~Hz})$ with a Finite Impulse Response filter and resampled at $125 \mathrm{~Hz}$. Signals containing noise were discarded after visual inspection.

The oscillatory activity recorded from the STN was quantified in the frequency domain by analyzing power spectral density (PSD) for the recorded LFPs in the two phases (hyperacute and chronic), using a non-parametric approach based on the discrete Fourier transform (DFT). More specifically, spectra were calculated using Welch's averaged, modified periodogram [26]. A Hanning window $W(j)$ was applied to divide the signal, containing $\mathrm{n}$ samples, into K segments and for each segment the modified periodogram $I_{k}$ was calculated from the DFT coefficients $A_{k}(n)$. Each segment was 128 samples in length $(L=128)$, to obtain a frequency resolution of $0.98 \mathrm{~Hz}$.

$$
\begin{aligned}
& A_{k}(n)=\frac{1}{L} \sum_{j=0}^{L-1} X_{k}(j) W(j) e^{-\frac{2 \pi i n j}{L}}, i=(-1)^{\frac{1}{2}} \\
& I_{k}\left(f_{n}\right)=\frac{L}{U}\left|A_{k}(n)\right|^{2}, \\
& K=1,2,3, \ldots, K, f_{n}=\frac{n}{L}, n=1,2,3, \ldots, \frac{L}{2}, U=\frac{1}{L} \sum_{j=0}^{L-1} W^{2}(j)
\end{aligned}
$$

The PSD of each signal was obtained by averaging the periodogram $I_{k}$ calculated in each segment. phase (immediately after surgery, $\mathrm{t}-0 \mathrm{~h})$ and the chronic phase (30 days after surgery, t-30d). The gray arrow represents time (days). The lower panel shows the three LFP recording conditions: before stimulation (baseline, $2 \mathrm{~min}$ ), during stimulation (DBS on, $6 \mathrm{~min}$ ) and after stimulation (DBS off, 2 min). The black line represents time (min).

To test whether DBS influenced LFP activity at both $\mathrm{t}-\mathrm{Oh}$ and $\mathrm{t}$-30d, we considered three states of interest: baseline (1 min preDBS), DBS on (1 min after DBS was turned on), and DBS off (1 min after DBS was turned off).

To account for the high inter-nuclei variability, PSD in each nucleus was normalized by the total spectral power in the 2-45 $\mathrm{Hz}$ frequency range during the baseline condition in hyperacute phase according to the following formula:

$$
\operatorname{PSD}_{N}(f)=\frac{\operatorname{PSD}(f)}{\sum_{f=2}^{45} P S D_{\text {baseline } \mathrm{t}-\mathrm{oh}}(f)}
$$

where $\mathrm{PSD}_{\mathrm{N}}$ is the normalized PSD, and $\mathrm{f}$ is the frequency.

LFP analysis focused on the beta frequency band detected in each phase for each nucleus as follows. Beta band was analyzed estimating the spectral power, calculated as:

$$
S P_{B}=\sum_{f \in B} P S D(f)
$$

where $\mathrm{B}$ is the beta band (see Identifying the Beta Band) and $S P_{B}$ is the spectral power.

In addition, to detect the time course of beta modulation throughout the whole experimental session, and to compare it with baseline, data were analyzed with a time-frequency approach (short-time Fourier transform, STFT). In each LFP recording, a time-moving Hanning window, $2.05 \mathrm{~s}$ long, was applied to the signal: the window was moved forward $1.02 \mathrm{~s}$ obtaining a recording segment $2.05 \mathrm{~s}$ long every $1.02 \mathrm{~s}$ advance and each segment overlapped the previous segment by $1.02 \mathrm{~s}$. The resulting time-frequency spectrum was calculated in each $2.05 \mathrm{~s}$ segment, with a final time resolution of $1.02 \mathrm{~s}$ and a frequency resolution of $0.49 \mathrm{~Hz}$.

Beta time course was determined from the time-frequency spectrum by calculating the integral in time of the specific beta band for each nucleus. Beta time course was studied as percentage power changes from baseline recording that was calculated as mean value of power during the 2 min before turning the DBS on. 
Table 3. Nuclei characterization during ongoing DBS in the 7 patients with PD

\begin{tabular}{|c|c|c|c|c|c|c|c|c|c|c|}
\hline \multirow[t]{2}{*}{ Patient } & \multicolumn{2}{|c|}{ Recording side } & \multicolumn{2}{|l|}{ Beta } & \multicolumn{2}{|c|}{ Frequency peak } & \multicolumn{2}{|c|}{ DBS ON } & \multicolumn{2}{|c|}{ DBS OFF } \\
\hline & $\mathrm{t}-0 \mathrm{~h}$ & $\mathrm{t}-30 \mathrm{~d}$ & $\mathrm{t}-0 \mathrm{~h}$ & $\mathrm{t}-30 \mathrm{~d}$ & $\mathrm{t}-\mathrm{Oh}$ & $t-30 d$ & $\mathrm{t}-0 \mathrm{~h}$ & $t-30 d$ & $\mathrm{t}-0 \mathrm{~h}$ & $t-30 d$ \\
\hline 2 & Right & & $x$ & $x$ & 17 & 15 & $\downarrow$ & $\downarrow$ & $\uparrow \uparrow$ & $\uparrow \uparrow$ \\
\hline 4 & Right & & $x$ & $x$ & 15 & 13 & $\downarrow$ & $\downarrow$ & $\uparrow$ & $\uparrow \uparrow$ \\
\hline 4 & Left & & $x$ & $\times$ & 9 & 11 & $\downarrow \downarrow$ & $\downarrow$ & $\uparrow \uparrow$ & $\uparrow$ \\
\hline 6 & Right & & $x$ & $x$ & 12 & 11 & $\downarrow$ & nd & $\uparrow$ & nd \\
\hline 6 & Left & & $x$ & $x$ & 10 & 17 & $\downarrow$ & $\downarrow$ & $\uparrow$ & $\uparrow$ \\
\hline 7 & Right & & $x$ & $x$ & 11 & 14 & $\downarrow$ & $\downarrow$ & $\uparrow$ & $\uparrow$ \\
\hline 7 & Left & & $x$ & $x$ & 13 & 12 & $\downarrow$ & $\downarrow$ & $\uparrow$ & $\uparrow$ \\
\hline 1 & Right & & - & - & - & - & $\uparrow$ & $\uparrow \uparrow$ & $\downarrow$ & $\downarrow \downarrow$ \\
\hline 1 & Left & & - & - & - & - & nd & nd & nd & nd \\
\hline 2 & Left & & - & - & - & - & $\uparrow \uparrow$ & $\uparrow \uparrow$ & $\downarrow$ & $\downarrow$ \\
\hline 3 & Right & & - & - & - & - & $\downarrow$ & $\uparrow$ & $\uparrow$ & $\uparrow$ \\
\hline 3 & Left & & - & - & - & - & $\uparrow \uparrow$ & $\uparrow \uparrow$ & $\downarrow$ & $\downarrow$ \\
\hline 5 & Right & & - & - & - & - & $\downarrow$ & nd & $\uparrow$ & nd \\
\hline
\end{tabular}

$\times=$ Significant beta band found; $\mathrm{nd}=$ not defined because signals contained noise. Arrows within DBS ON column indicate the qualitative increase or decrease of beta band in each nucleus compared with baseline ( $\downarrow$ decrease of beta band $<50 \%$ compared to baseline; $\downarrow \downarrow$ decrease of beta band $\geq 50 \%$ compared to baseline; $\uparrow$ increase of beta band $<50 \%$ compared to baseline; $\uparrow \uparrow$ increase of beta band $\geq 50 \%$ compared to baseline); arrows within DBS OFF column indicate the increase or decrease compared with DBS ON.

To analyze the beta time course (excluding the baseline) during the conditions DBS on and DBS off, we subdivided the time recording in consecutive segments 30 s long starting from 0 , yielding 12 segments in the DBS on condition (from ON1 to ON12) and 4 segments in the DBS off condition (from OFF1 to OFF4). We also compared mean value for segments in the hyperacute and chronic phases.

\section{Identifying the Beta Band}

Each nucleus was characterized to detect the beta range and its corresponding peak. To do so, the mean and standard deviation (SD) of PSD in the $8-45 \mathrm{~Hz}$ band in each nucleus were considered to obtain the threshold for significant spectral bands in the hyperacute and chronic phases (95\% confidence interval (CI): mean $\pm 1.97 \mathrm{SD})$. The beta activity was considered significant if PSD values in the $8-30 \mathrm{~Hz}$ range exceeded the threshold. If the beta activity was significant, we defined the maximum PSD value within the $8-30 \mathrm{~Hz}$ range as the beta peak. We then considered the frequency band ranging $\pm 4 \mathrm{~Hz}$ around the beta peak for subsequent analyses. If beta activity was not significant we considered a standard beta frequency range. The individual beta band was classified as low-beta band if the beta peak found belonged to the $8-20 \mathrm{~Hz}$ band and as high-beta band if the peak found belonged to the $21-30 \mathrm{~Hz}$ band. To account for the inter-nuclei variability, the spectral power in the beta band was normalized by the length of the beta band in the corresponding phase.

\section{Statistical Analysis}

To evaluate the effect of time on macroelectrode impedance, a two-tailed paired Student $t$ test was used to compare all values for the contact pairs of macroelecrode used for recording in hyper- acute and chronic phases. To assess the dependency of beta on the impedance, Pearson's linear correlation coefficient (GraphPad Prism 3.0) was calculated $(\mathrm{p}<0.05)$ between impedance values in both phases and individual beta powers.

Two-tailed paired Student's t test was also used to evaluate the effect of time on central frequency of the beta peak: values were compared in the hyperacute and chronic phases.

To evaluate the correlation between nuclei with significant beta band activity and the contact pairs used for recording, we performed a Pearson's $\chi^{2}$ test. A one-way repeated measures analysis of variance (ANOVA) (STATISTICA 5.5; StatSoft Inc.) was used to test differences between each target coordinate of macroelectrodes $(\mathrm{X}, \mathrm{Y}, \mathrm{Z})$ (table 2) in nuclei with and without significant beta band activity with factor 'beta band' (2 levels: presence, absence). To study the effect of DBS on LFP activity, data were analyzed for the whole population and considering only the nuclei with significant beta band activity. A two-way ANOVA for beta spectral power was run with factors 'stimulation state' (within factor, 3 levels: baseline, DBS on, DBS off) and 'recording phase' (within factor, 2 levels: hyperacute and chronic). Tukey honest significant test was used for post hoc analysis; differences were considered significant at $\mathrm{p}<0.05$.

To analyze the statistical significance of data for nuclei with a significant beta band and to evaluate the effect of stimulation over time, we tested the percentage power changes in beta power through change point analysis with a percentage time resolution of $3.3 \%$ [27]. Change was significant at a $95 \%$ confidence level $(\mathrm{p}<0.05)$. The time course of beta activity in the hyperacute and chronic phases was compared in DBS on and DBS off conditions. To study the DBS on condition a two-way ANOVA with factor 'time' (within factor, DBS on: 12 levels from ON1 to ON12) and 

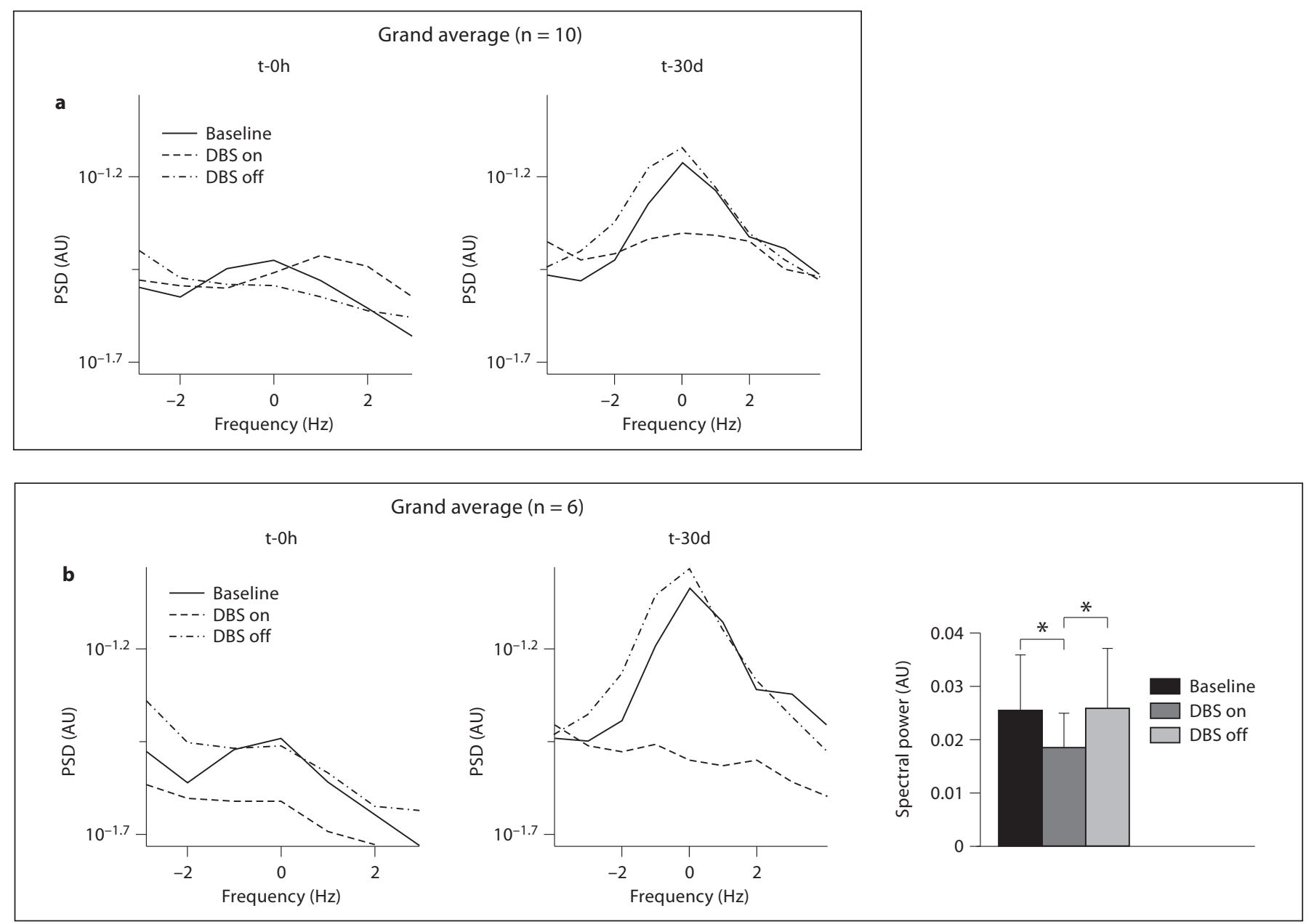

Fig. 2. Spectral analysis. a Hyperacute (t-0h) and chronic (t-30d) phases for whole population: grand average for whole population ( $\mathrm{n}=10$ nuclei) of the power spectral density (PSD) of beta band in the three experimental conditions: pre-stimulation (baseline; ), during stimulation (DBS on; ------) and after stimulation (DBS off; -.-.-.---) in the t-0h phase on the left and in the t-30d phase on the right. Each PSD was normalized by the total spectral power in the $2-45 \mathrm{~Hz}$ frequency range of hyperacute baseline. Beta band PSD was aligned on beta peak of each nucleus $(0 \mathrm{~Hz})$; if peak was not found, PSD was aligned on central value of standard low-beta band $(9-21 \mathrm{~Hz})$. On the y-axis the normalized PSD (arbitrary units (AU), logarithmic scale) and on the $\mathrm{x}$-axis the frequency $(\mathrm{Hz}$, linear scale). b t- $0 \mathrm{~h}$ and $\mathrm{t}-30 \mathrm{~d}$ phases for nuclei with significant beta band: grand average for nuclei with signifi-

factor 'recording phase' (within factor, 2 levels: hyperacute and chronic) was used. To study the DBS off condition we used a twoway ANOVA with factor 'time' (within factor, DBS off: 4 levels from OFF1 to OFF4) and factor 'recording phase' (within factor, 2 levels: hyperacute and chronic). Tukey honest significant test was used for post hoc analysis; differences were considered significant at $\mathrm{p}<0.05$. cant beta band ( $\mathrm{n}=6$ nuclei) of PSD of beta band in the three experimental conditions: pre-stimulation (baseline; - - ) during stimulation (DBS on; ------) and after stimulation (DBS off; -.-.-.-.-) in the $\mathrm{t}-0 \mathrm{~h}$ and in the $\mathrm{t}-30 \mathrm{~d}$ phases. The plot is organized as in $\mathbf{a}$. The histogram represents beta power for nuclei with significant beta band in the $\mathrm{t}-0 \mathrm{~h}$ and the $\mathrm{t}-30 \mathrm{~d}$ phases grouped together for each condition (black = baseline; gray = DBS on; light gray $=$ DBS off). Error bars represent the standard deviation (SD). On the $y$-axis the normalized power of beta band (AU) and on the $\mathrm{x}$-axis the three conditions. ${ }^{*} \mathrm{p}<0.05$, post hoc two-way repeated measures analysis of variance (ANOVA). Note that beta band power decreases in on DBS condition both in hyperacute and chronic phases for nuclei with significant beta band.

\section{Results}

Confirming previous results [22], in all 7 patients macroelectrode impedance changed significantly over time (mean $\pm \mathrm{SD}$; $\mathrm{t}-0 \mathrm{~h}$ vs. $\mathrm{t}-30 \mathrm{~d}$ : $15.33 \pm 4.59$ vs. $9.15 \pm 1.97$ $\mathrm{K} \Omega, \mathrm{p}=0.005$ ) (table 2). Correlation analysis between 


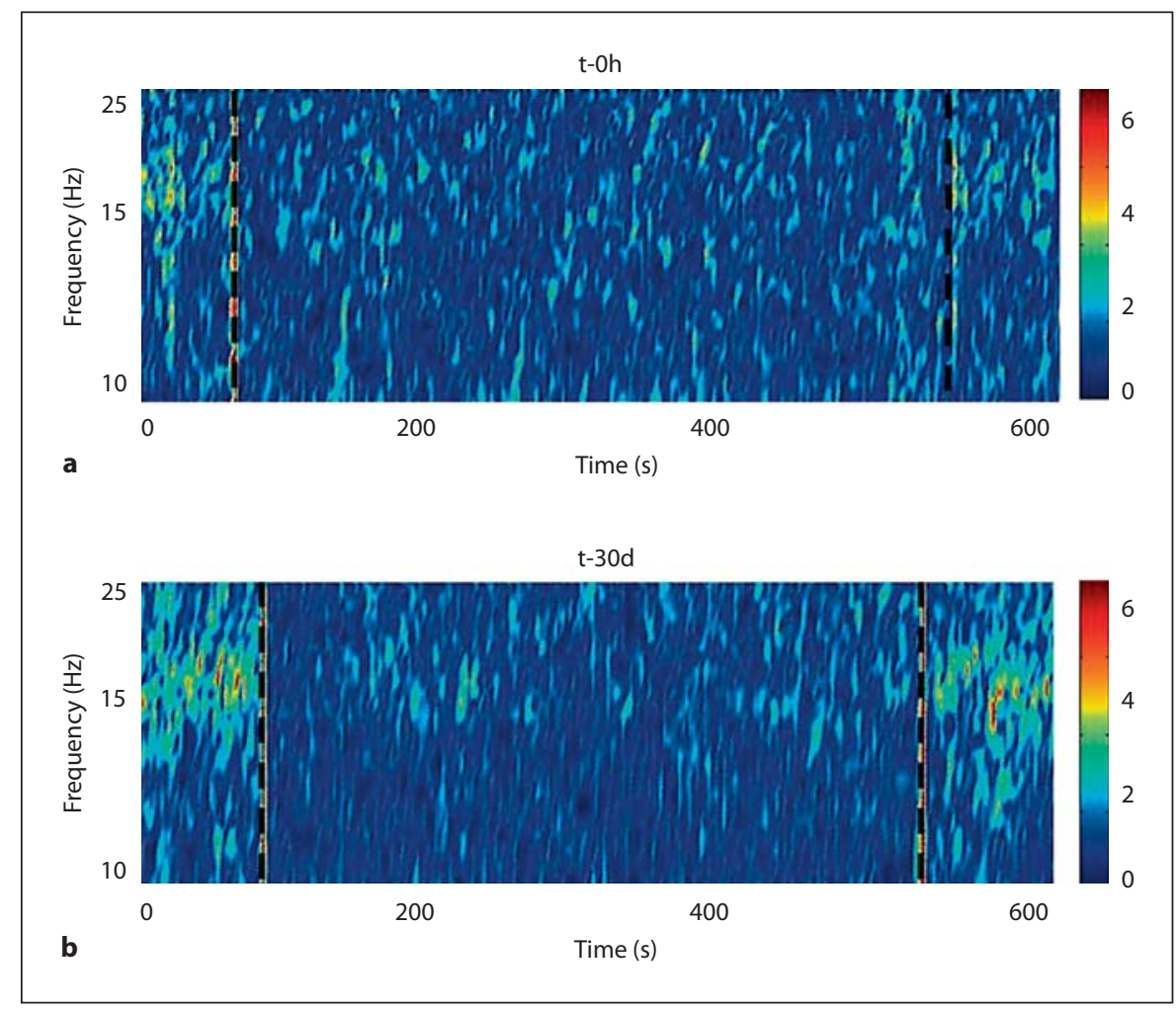

Fig. 3. Time-frequency plots of beta power in a single representative nucleus. Subthalamic nucleus-local field potential (STN-LFP) time varying power spectrum in the beta band of right nucleus of patient 2. a Time-frequency plot over the entire experimental in hyperacute phase $(\mathrm{t}-0 \mathrm{~h})$. The black dashed lines, from the left, correspond to turning DBS on and to turning DBS off, respectively. Temporal resolution: $1.02 \mathrm{~s}$; frequency resolution: $0.46 \mathrm{~Hz}$. On the $y$-axis the frequency of LFP oscillations in the beta range (10-25 $\mathrm{Hz}, \log$ scale) and on $\mathrm{x}$-axis the time (seconds, linear scale). Oscillation power is color-coded on the bar on the right (red: high power; blue: low power). b Time-frequency plot over the entire experiment in chronic phase (t-30d). The plot is organized as in a. Note that beta band power decreased more prominently in chronic phase than in the hyperacute phase.

Owing to artifacts due to electrical devices of the operating room, we excluded three nuclei from the analysis (table 3). The subsequent analysis was conducted on a total of 10 nuclei, of which 6 showed a significant beta peak. Data for the whole population ( $\mathrm{n}=10$ nuclei) failed to find significant changes in beta spectral power during DBS at $\mathrm{t}-0 \mathrm{~h}$ and t-30d (fig. 2a). Conversely, power spectra for nuclei with significant beta band activity ( $\mathrm{n}=6$ nuclei) showed significant changes in beta activity during DBS in both recording phases (fig. 2b). Two-way repeated measures ANOVA disclosed a significant effect of the factor 'stimulation state' $(p=0.016)$, whereas no effects either of the factor 'recording phase' or of the interaction between the two factors was found. Post hoc analysis for the factor 'stimulation state' showed that beta band power significantly decreased during stimulation $(\mathrm{p}=0.021)$ and significantly increased when DBS was turned off $(p=0.037)$ returning to baseline value (fig. $2 b$ ). 


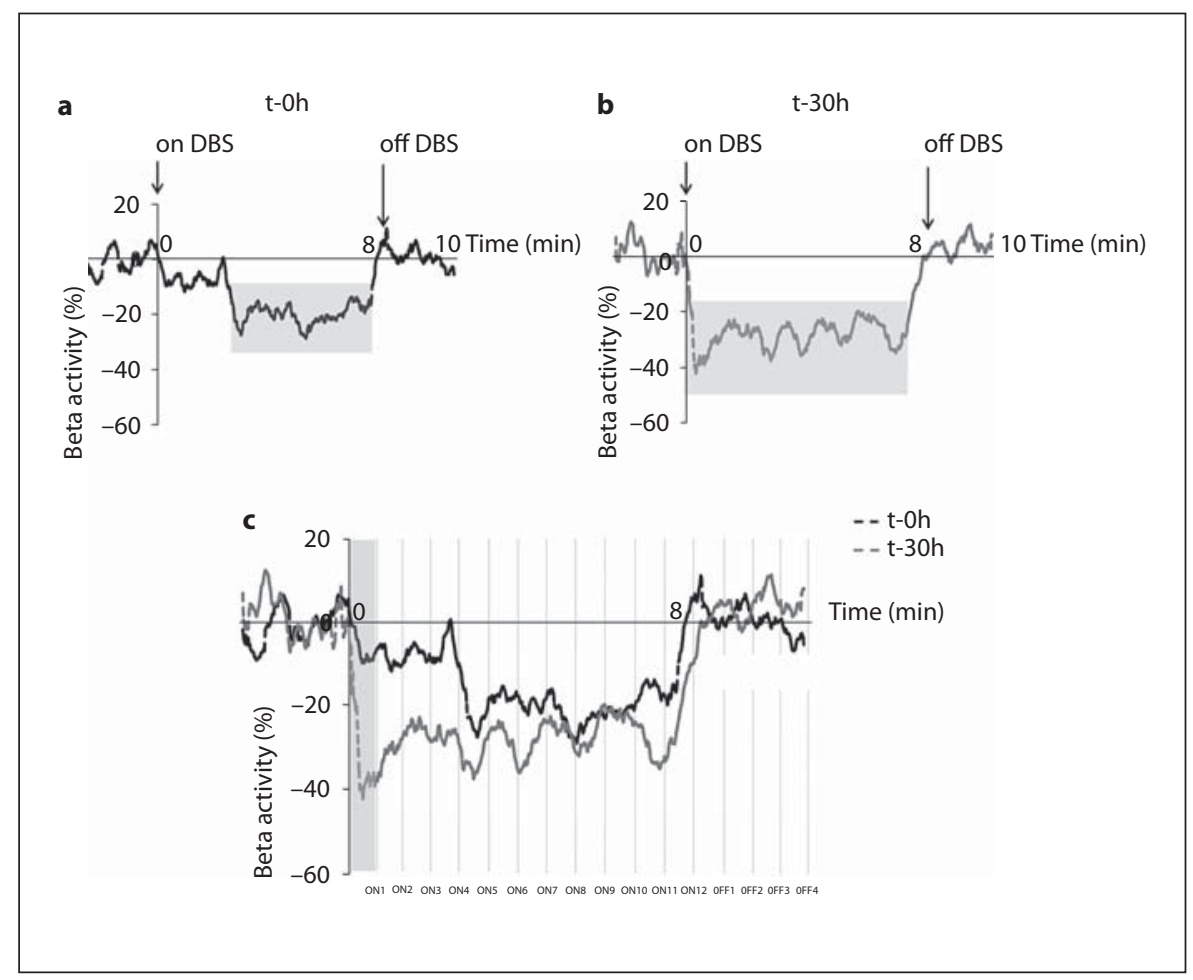

Fig. 4. Time course of beta activity. Beta power modulation during experimental session in hyperacute $(\mathrm{t}-\mathrm{Oh})$ and chronic phases $(\mathrm{t}-$ $30 \mathrm{~d}$ ). a Grand average ( $\mathrm{n}=6$ nuclei) of the beta activity expressed as percentage power change from the baseline in the $\mathrm{t}-0 \mathrm{~h}$ phase. On the $y$-axis are represented beta power (\%) and on $\mathrm{x}$-axis the time (min). Gray area represents a significant beta power change $(\mathrm{p}<0.05)$. $\mathbf{b}$ Grand average $(n=6$ nuclei) of the beta activity expressed as percentage power change from the baseline in the $t-30 \mathrm{~d}$ phase. The plot is organized as in a. c Grand average ( $\mathrm{n}=6$ nuclei) of the beta activity expressed as percentage power change from

Figure 3 represents a case of beta time course during the two experimental sessions ( $\mathrm{t}-0 \mathrm{~h}, \mathrm{t}-30 \mathrm{~d})$.

In the analysis focusing on nuclei with a significant beta band at baseline, averaged data in the $\mathrm{t}-0 \mathrm{~h}$ and $\mathrm{t}-30 \mathrm{~d}$ phases showed significant beta desynchronization when DBS was turned on. In $\mathrm{t}-0 \mathrm{~h}$, change-point analysis detected a significant beta band decrease (from -7.96 to $-20.16 \%$ from baseline, confidence level $=100 \%$ ) after 2.44 min while DBS was turned on. When DBS was turned off, beta band power immediately increased (from -20.16 to $3.09 \%$ from baseline, confidence level $=100 \%$ ) (fig. 4a). Similarly, in t-30d, when DBS was turned on, beta power immediately decreased (from 18.06 to $-29.32 \%$ from baseline, confidence level $=100 \%$ ) and when DBS was turned off increased (from -29.32 to $3.88 \%$ from baseline, confidence level $=99 \%$ ) (fig. $4 \mathrm{~b}$ ). the baseline in the t-0h phase (black) and t-30d phase (dark gray). The gray vertical lines, from the left, correspond to the 12 segments of DBS on condition (ON1 to ON12) and the 4 segments of DBS off condition (OFF1 to OFF4) respectively. The plot is organized as in $\mathbf{a}$. The gray area represents the significant difference between hyperacute and chronic beta activity $(\mathrm{p}<0.05)$. Note that both averaged data in hyperacute and chronic phases showed significant beta desynchronization when DBS was turned on and beta change was significantly higher in chronic than in the hyperacute phase in the first segment.

The time course of beta activity during DBS on condition differed significantly between $\mathrm{t}-\mathrm{h}$ and $\mathrm{t}-30 \mathrm{~d}$ (ANO$V A$, interaction 'time' $\times$ 'recording phase' $p=0.014$ ). Post hoc analysis showed more significant beta activity changes from baseline in the $\mathrm{t}-30 \mathrm{~d}$ phase than in the $\mathrm{t}-0 \mathrm{~h}$ phase in the first segment: ON1 (t-0h vs. t-30d: $-7.59 \pm 3.36$ vs. $-42.53 \pm 9.08 \%, p=0.0003)$. Conversely, ANOVA failed to find changes in DBS off condition beta activity between the hyperacute and chronic phases (fig. 4c).

\section{Discussion}

Our results showed a stationary intra-nuclear STN beta band LFP activity in recordings obtained immediately after DBS surgery (hyperacute phase) and 30 days 
after surgery (chronic phase) confirming that beta power peaks maintain at long-term recording. Although only in nuclei showing a prominent beta activity at baseline $(54 \%$ of sample), STN DBS markedly reduced beta LFP oscillations during both hyperacute and chronic phases, it induced the greatest reduction during chronic phase. The new evidence we provided describing STN DBS induced changes in STN beta LFPs during DBS in chronic phase offers essential information needed to develop a new adaptive DBS system.

Because LFP beta oscillations varied widely among the studied STN nuclei, beta activity exceeded threshold values in only in 7 of the 13 STN nuclei studied (54\%). This finding agrees with previous data reporting similar beta variability within the population studied (67 and 68\%) $[10,12]$. The slight difference in our population was likely due to a gender effect (6 men and 1 woman) given that beta band power is lower in males.

The beta activity observed was then characterized defining the highest peak within the band, in order to identify a possible marker of DBS action. We observed that, if the beta activity was significant, the highest oscillation was in the low-beta band and was then disrupted by DBS. In our sample, the significant beta activity was never characterized by a high-beta peak. This finding is in line with previous studies suggesting that, in the off medication state, the high-beta peak was shown to be lower than and non-linearly dependent on the low-beta peak [28]. Hence, because levodopa and DBS specifically act on the low-beta activity, our results support the hypothesis that the low-beta rhythm can be considered as a marker of pathology whereas the high-beta rhythm can be essentially a physiological rhythm $[3,5,28]$.

Nuclei characterization therefore showed stationary intra-nuclear beta activity from the hyperacute to the chronic recording phase (7 nuclei had a significant beta peak at baseline under both experimental phases and 6 nuclei did not have a beta peak in both phases). This marked stationarity in intra-nuclear beta band activity suggests that, at least in a fraction of nuclei, a specific rhythm exists and may be a parkinsonian patient's 'signature'. Various studies have shown abnormal synchronized oscillatory activity in the STN and internal segment of the globus pallidus (GPi) of MPTP-lesioned non-human primates and patients with $\mathrm{PD}$, showing that it is further related to symptoms of illness and is an important feature of PD [1, 2, 8, 19, 29-36], including the abnormal beta activity [10, 37-40].

Given that we found no correlation between beta power and recording macroelectrode impedance, we confirmed previous evidence [22] that macroelectrode im- pedance changes occurring between the two recording phases have no effect on beta band power stationarity. An interesting finding that confirms current knowledge on beta activity was that LFP from nuclei having a significant beta peak were all recorded bilaterally from the uppermost contacts (1-3). This finding fits in with previous observations that beta activity in the LFP tends to be greater in the dorsolateral than in ventromedial STN $[41,42]$. Considering the STN target for PD, the dorsolateral portion could be the most effective area of STN that seems to be involved in sensorimotor circuits [43-45]. The most effective electrode contact lies in the upper part of the STN intraoperative recording area (near the dorsal border) confirming that this region has a key role in the clinical effectiveness of the STN DBS $[44,46,47]$. A recent study showed a correlation between the depth where the beta activity recorded intraoperatively was highest (local beta generator) and the depth of the contact independently chosen for chronic DBS [48]. Hence, if we think that a local beta generator exists, then nuclei having low-beta activity could be far from the beta generator, whereas nuclei with a significant beta peak could be close to the beta generator. Notwithstanding this possibility, some investigators noted that although the electrode position failed to limit the beneficial effect of chronic DBS, adjusting the stimulation intensity yielded benefits in all patients [49]. In particular, if the depth of contact for chronic DBS is far from depth of beta generator, more voltage has to be delivered [48]. Since we used the same recording contacts in the hyperacute and chronic phases, the recording coordinates remained constant, thus supporting the stationary beta activity.

The previous considerations are valid for the nuclei in which beta activity is evident. There is a percentage of nuclei in which this activity is not evident independently from the position of the macroelectrodes [10]. Since the clinical effects of DBS in patients with a marked beta activity is not different from the one observed in subjects that do not have this characteristic, only in some patients beta activity can be considered one of the reliable marker of PD. Other bands may represent a possible marker in other patients. Previous literature showed low-frequency LFP modulations after drug administration $[3,28]$ and during STN stimulation [16]. The authors found that these changes were correlated with the parkinsonian patients' clinical features.

Our study leaves open the question how STN DBS acts on the various STN nuclei in patients with PD. When we turned DBS on, STN LFP beta oscillations decreased only in those nuclei with higher beta activity at baseline and when we turned DBS off, the DBS-induced beta band 
suppression disappeared and the activity patterns recorded at baseline returned $[5,10]$. In a previous study conducted in our laboratory, considering the whole population, we failed to show significant LFP beta power suppression during DBS [16], presumably because not all recordings had abnormal peaks in the beta band before DBS and because some patients were on dopaminergic medication, a condition known to decrease beta activity. More recent studies reported that the beta oscillations decreased significantly during DBS but did so only in patients whose LFP recordings already showed high-beta activity at baseline $[10,12]$.

A distinctive point in this study is that whereas previous studies investigated the neurophysiologic effects of STN DBS within days after macroelectrode implantation, we studied them also in patients with PD undergoing DBS 30 days later. We found that in nuclei with high-beta activity at baseline, DBS reduced beta activity immediately after surgery and 30 days later. This finding suggests that the results of LFP recordings obtained in the hyperacute phase could be extended to the chronic phase although DBS markedly reduced STN beta LFPs in the chronic phase. Trauma from macroelectrode penetration causes a micro-subthalamotomic effect that transiently mimics the levodopa-induced effect [3] and is accompanied by a transient hyperacute improvement in parkinsonian motor symptoms [50]. In agreement with this hypothesis that takes into account possible changes in the STN networks due to plasticity mechanisms after macroelectrode implantation, the greater beta reduction we observed in the chronic phase could reflect the re-established pathological pattern.

Our finding that the DBS-induced local beta activity suppression, when present, remained stable in time, raises the possibility that the reduced beta activity underlies some of the therapeutic actions of DBS [10, 12, 40]. Some studies suggest that STN DBS could produce its clinical benefits by disrupting pathological oscillations at beta or lower frequencies also in the GPi $[18,51]$ as well as in the substantia nigra pars reticulata $(\mathrm{SNr})[52,53]$. Besides, since striatal dopamine release during high-frequency stimulation has been observed [54,55], we could hypothesize that disrupting pathological oscillation effect could mimic the disruption of beta pathological oscillation induced by levodopa as seen in previous studies $[3,12,28]$. Moreover, STN DBS could produce its clinical effects by artificially mimicking the pacing action of the $300-\mathrm{Hz}$ subthalamic rhythm which is lost in PD $[56,57]$. In neurophysiological study, STN DBS activated not only the STN and its direct efferents but also the thalamus, the basal ganglia, the somatosensory cortex, the motor cortex, and the supplementary motor area as disclosed also by functional imaging $[58,59]$. DBS generates efferent output transmitted to non-stimulated nuclei, supporting the hypothesis that STN-DBS acts in the entire cortexbasal ganglia-cortex network [13, 18, 60-62].

Our study describing neurosignals also provides the essential information we need for developing a new adaptive DBS system $[14,16,21,22]$. Because STN LFPs can be directly recorded from the implanted macroelectrodes also 30 days after surgery, STN oscillatory activity could be used in a novel closed-loop DBS system able to change stimulation variables automatically adapting them to the patient's clinical state $[12,14]$. The idea of controlling DBS online through LFPs implies the identification of robust variables able to describe patient's pathophysiological state and the DBS-induced changes in the STN LFP activity. Our study provides direct evidence that in selected patients with PD, STN beta activity could be a robust variable due to its stability and its DBS induced reduction in the hyperacute and chronic phases to reflect the patient's clinical status. What remains unclear is the possible correlation between beta band activity in patients undergoing DBS in chronic phase and the specific motor symptoms of PD. Since only a portion of our population showed a significant beta activity, we believe that other frequency bands, such as low-frequency band, could be used as variables of PD to adapt the stimulation parameters.

\section{Acknowledgments}

This study was supported by the ERANET-Neuron Grant 'PhysiolDBS' (Neuron-48-013), by Fondazione IRCCS Ca' Granda, Ospedale Maggiore Policlinico (Milan, Italy), Università degli Studi di Milano (Italy), Ministero della Sanità (Italy), Ministero dell'Università e della Ricerca Scientifica e Tecnologica (Italy).

\section{Disclosure Statement}

Sara Marceglia, Sergio Barbieri and Alberto Priori are stakeholders of Newronika SrL, a spin-off company of the Fondazione IRCCS Ca' Granda Ospedale Maggiore Policlinico of Milan and of the Università degli Studi di Milano. 


\section{References}

1 Brown P: Oscillatory nature of human basal ganglia activity: relationship to the pathophysiology of Parkinson's disease. Mov Disord 2003;18:357-363.

2 Levy R, Hutchison WD, Lozano AM, Dostrovsky JO: Synchronized neuronal discharge in the basal ganglia of parkinsonian patients is limited to oscillatory activity. J Neurosci 2002;22:2855-2861.

- 3 Priori A, Foffani G, Pesenti A, Tamma F, Bianchi AM, Pellegrini M, Locatelli M, Moxon KA, Villani RM: Rhythm-specific pharmacological modulation of subthalamic activity in Parkinson's disease. Exp Neurol 2004;189:369-379.

-4 Brucke C, Kupsch A, Schneider GH, Hariz MI, Nuttin B, Kopp U, Kempf F, Trottenberg T, Doyle L, Chen CC, Yarrow K, Brown P, Kuhn AA: The subthalamic region is activated during valence-related emotional processing in patients with Parkinson's disease. Eur J Neurosci 2007;26:767-774.

5 Foffani G, Bianchi AM, Baselli G, Priori A: Movement-related frequency modulation of beta oscillatory activity in the human subthalamic nucleus. J Physiol 2005;568:699711.

-6 Kuhn AA, Doyle L, Pogosyan A, Yarrow K, Kupsch A, Schneider GH, Hariz MI, Trottenberg T, Brown P: Modulation of beta oscillations in the subthalamic area during motor imagery in Parkinson's disease. Brain 2006; 129:695-706.

7 Kuhn AA, Hariz MI, Silberstein P, Tisch S, Kupsch A, Schneider GH, Limousin-Dowsey P, Yarrow K, Brown P: Activation of the subthalamic region during emotional processing in Parkinson disease. Neurology 2005; 65:707-713.

$>8$ Levy R, Ashby P, Hutchison WD, Lang AE, Lozano AM, Dostrovsky JO: Dependence of subthalamic nucleus oscillations on movement and dopamine in Parkinson's disease. Brain 2002;125:1196-1209.

-9 Marceglia S, Fiorio M, Foffani G, MrakicSposta S, Tiriticco M, Locatelli M, Caputo E, Tinazzi M, Priori A: Modulation of beta oscillations in the subthalamic area during action observation in Parkinson's disease. Neuroscience 2009;161:1027-1036.

10 Eusebio A, Thevathasan W, Doyle Gaynor L, Pogosyan A, Bye E, Foltynie T, Zrinzo L, Ashkan K, Aziz T, Brown P: Deep brain stimulation can suppress pathological synchronisation in parkinsonian patients. J Neurol Neurosurg Psychiatry 2011;82:569-573.

- 11 Foffani G, Ardolino G, Egidi M, Caputo E, Bossi B, Priori A: Subthalamic oscillatory activities at beta or higher frequency do not change after high-frequency DBS in Parkinson's disease. Brain Res Bull 2006;69:123130.

-12 Giannicola G, Marceglia S, Rossi L, MrakicSposta S, Rampini P, Tamma F, Cogiamanian F, Barbieri S, Priori A: The effects of levodopa and ongoing deep brain stimulation on subthalamic beta oscillations in Parkinson's disease. Exp Neurol 2010;226:120127.

13 Kuhn AA, Kempf F, Brucke C, Gaynor Doyle L, Martinez-Torres I, Pogosyan A, Trottenberg T, Kupsch A, Schneider GH, Hariz MI, Vandenberghe W, Nuttin B, Brown P: Highfrequency stimulation of the subthalamic nucleus suppresses oscillatory beta activity in patients with Parkinson's disease in parallel with improvement in motor performance. J Neurosci 2008;28:6165-6173.

14 Marceglia S, Rossi L, Foffani G, Bianchi A, Cerutti S, Priori A: Basal ganglia local field potentials: applications in the development of new deep brain stimulation devices for movement disorders. Expert Rev Med Devices 2007;4:605-614.

15 Priori A, Ardolino G, Marceglia S, MrakicSposta S, Locatelli M, Tamma F, Rossi L, Foffani G: Low-frequency subthalamic oscillations increase after deep brain stimulation in Parkinson's disease. Brain Res Bull 2006;71: 149-154.

16 Rossi L, Marceglia S, Foffani G, Cogiamanian F, Tamma F, Rampini P, Barbieri S, Bracchi F, Priori A: Subthalamic local field potential oscillations during ongoing deep brain stimulation in Parkinson's disease. Brain Res Bull 2008;76:512-521.

-17 Bronte-Stewart H, Barberini C, Koop MM, Hill BC, Henderson JM, Wingeier B: The STN beta-band profile in Parkinson's disease is stationary and shows prolonged attenuation after deep brain stimulation. Exp Neurol 2009;215:20-28.

18 Brown P, Mazzone P, Oliviero A, Altibrandi MG, Pilato F, Tonali PA, Di Lazzaro V: Effects of stimulation of the subthalamic area on oscillatory pallidal activity in Parkinson's disease. Exp Neurol 2004; 188:480-490.

19 Silberstein P, Kuhn AA, Kupsch A, Trottenberg T, Krauss JK, Wohrle JC, Mazzone P, Insola A, Di Lazzaro V, Oliviero A, Aziz T, Brown P: Patterning of globus pallidus local field potentials differs between Parkinson's disease and dystonia. Brain 2003;126:25972608.

20 Wingeier B, Tcheng T, Koop MM, Hill BC, Heit G, Bronte-Stewart HM: Intra-operative STN DBS attenuates the prominent beta rhythm in the STN in Parkinson's disease. Exp Neurol 2006;197:244-251.

21 Rossi L, Foffani G, Marceglia S, Bracchi F, Barbieri S, Priori A: An electronic device for artefact suppression in human local field potential recordings during deep brain stimulation. J Neural Eng 2007;4:96-106.

22 Rosa M, Marceglia S, Servello D, Foffani G, Rossi L, Sassi M, Mrakic-Sposta S, Zangaglia R, Pacchetti C, Porta M, Priori A: Time-dependent subthalamic local field potential changes after DBS surgery in Parkinson's disease. Exp Neurol 2010;222:184-190.
23 LIMPE: Guidelines for the treatment of Parkinson's disease. Neurol Sci 2003;24:s203s204.

24 Marceglia S, Mrakic-Sposta S, Tommasi G, Bartolomei L, Foresti C, Valzania F, Galati S, Stefani A, Tamma F, Priori A: Multicenter study report: electrophysiological monitoring procedures for subthalamic deep brain stimulation surgery in Parkinson's disease. Neurol Sci 2010;31:449-457.

25 Egidi M, Rampini P, Locatelli M, Farabola M, Priori A, Pesenti A, Tamma F, Caputo E, Chiesa V, Villani RM: Visualisation of the subthalamic nucleus: a multiple sequential image fusion (MUSIF) technique for direct stereotaxic localisation and postoperative control. Neurol Sci 2002;23(suppl 2):S71S72.

26 Welch P: The use of fast Fourier transform for the estimation of power spectra: a method based on time averaging over short, modified periodograms. IEEE Trans Acoust 1967;15:70-73.

27 Chen G, Gupta A: Parametric Statistical Change Point Analysis. Boston, Birkhauser, 2000.

28 Marceglia S, Foffani G, Bianchi AM, Baselli G, Tamma F, Egidi M, Priori A: Dopaminedependent non-linear correlation between subthalamic rhythms in Parkinson's disease. J Physiol 2006;571:579-591.

29 Bergman H, Wichmann T, Karmon B, DeLong MR: The primate subthalamic nucleus. II. Neuronal activity in the MPTP model of parkinsonism. J Neurophysiol 1994;72:507520.

- 30 Brown P, Oliviero A, Mazzone P, Insola A, Tonali P, Di Lazzaro V: Dopamine dependency of oscillations between subthalamic nucleus and pallidum in Parkinson's disease. J Neurosci 2001;21:1033-1038.

-31 Levy R, Hutchison WD, Lozano AM, Dostrovsky JO: High-frequency synchronization of neuronal activity in the subthalamic nucleus of parkinsonian patients with limb tremor. J Neurosci 2000;20:7766-7775.

- 32 Nini A, Feingold A, Slovin H, Bergman H: Neurons in the globus pallidus do not show correlated activity in the normal monkey, but phase-locked oscillations appear in the MPTP model of parkinsonism. J Neurophysiol 1995;74:1800-1805.

33 Raz A, Vaadia E, Bergman H: Firing patterns and correlations of spontaneous discharge of pallidal neurons in the normal and the tremulous 1-methyl-4-phenyl-1,2,3,6-tetrahydropyridine vervet model of parkinsonism. J Neurosci 2000;20:8559-8571.

34 Williams D, Tijssen M, Van Bruggen G, Bosch A, Insola A, Di Lazzaro V, Mazzone P, Oliviero A, Quartarone A, Speelman H, Brown P: Dopamine-dependent changes in the functional connectivity between basal ganglia and cerebral cortex in humans. Brain 2002;125:1558-1569. 
-35 Kuhn AA, Kupsch A, Schneider GH, Brown P: Reduction in subthalamic $8-35 \mathrm{~Hz}$ oscillatory activity correlates with clinical improvement in Parkinson's disease. Eur J Neurosci 2006;23:1956-1960.

- 36 Kuhn AA, Williams D, Kupsch A, Limousin P, Hariz M, Schneider GH, Yarrow K, Brown P: Event-related beta desynchronization in human subthalamic nucleus correlates with motor performance. Brain 2004;127:735746.

37 Brown P, Williams D: Basal ganglia local field potential activity: character and functional significance in the human. Clin Neurophysiol 2005;116:2510-2519.

38 Chen CC, Hsu YT, Chan HL, Chiou SM, Tu PH, Lee ST, Tsai CH, Lu CS, Brown P: Complexity of subthalamic $13-35 \mathrm{~Hz}$ oscillatory activity directly correlates with clinical impairment in patients with Parkinson's disease. Exp Neurol 2010;224:234-240.

- 39 Hammond C, Bergman H, Brown P: Pathological synchronization in Parkinson's disease: networks, models and treatments. Trends Neurosci 2007;30:357-364.

-40 Kuhn AA, Tsui A, Aziz T, Ray N, Brucke C, Kupsch A, Schneider GH, Brown P: Pathological synchronisation in the subthalamic nucleus of patients with Parkinson's disease relates to both bradykinesia and rigidity. Exp Neurol 2009;215:380-387.

-41 Chen CC, Pogosyan A, Zrinzo LU, Tisch S, Limousin P, Ashkan K, Yousry T, Hariz MI, Brown P: Intra-operative recordings of local field potentials can help localize the subthalamic nucleus in Parkinson's disease surgery. Exp Neurol 2006;198:214-221.

$\checkmark 42$ Kuhn AA, Trottenberg T, Kivi A, Kupsch A, Schneider GH, Brown P: The relationship between local field potential and neuronal discharge in the subthalamic nucleus of patients with Parkinson's disease. Exp Neurol 2005; 194:212-220.

43 Hamel W, Fietzek U, Morsnowski A, Schrader B, Herzog J, Weinert D, Pfister G, Muller D, Volkmann J, Deuschl G, Mehdorn HM: Deep brain stimulation of the subthalamic nucleus in Parkinson's disease: evaluation of active electrode contacts. J Neurol Neurosurg Psychiatry 2003;74:1036-1046.
44 Lanotte MM, Rizzone M, Bergamasco B, Faccani G, Melcarne A, Lopiano L: Deep brain stimulation of the subthalamic nucleus: anatomical, neurophysiological, and outcome correlations with the effects of stimulation. J Neurol Neurosurg Psychiatry 2002; 72:53-58.

45 Zheng Z, Zhang Y, Li J, Zhang X, Zhuang P, Li Y: Subthalamic deep brain stimulation for Parkinson's disease: correlation of active contacts and electrophysiologically mapped subthalamic nucleus. Chin Med J 2009;122: 2419-2422.

46 Maks CB, Butson CR, Walter BL, Vitek JL, McIntyre CC: Deep brain stimulation acti vation volumes and their association with neurophysiological mapping and therapeutic outcomes. J Neurol Neurosurg Psychiatry 2009;80:659-666.

47 Saint-Cyr JA, Hoque T, Pereira LC, Dostrovsky JO, Hutchison WD, Mikulis DJ, Abosch A, Sime E, Lang AE, Lozano AM: Localization of clinically effective stimulating electrodes in the human subthalamic nucleus on magnetic resonance imaging. J Neurosurg 2002;97:1152-1166.

48 Yoshida F, Martinez-Torres I, Pogosyan A, Holl E, Petersen E, Chen CC, Foltynie T, Limousin P, Zrinzo LU, Hariz MI, Brown P: Value of subthalamic nucleus local field potentials recordings in predicting stimulation parameters for deep brain stimulation in Parkinson's disease. J Neurol Neurosurg Psychiatry 2010;81:885-889.

49 Moro E, Esselink RJ, Xie J, Hommel M, Benabid AL, Pollak P: The impact on Parkinson's disease of electrical parameter settings in STN stimulation. Neurology 2002;59:706713.

50 Deuschl G, Herzog J, Kleiner-Fisman G, Kubu C, Lozano AM, Lyons KE, RodriguezOroz MC, Tamma F, Troster AI, Vitek JL, Volkmann J, Voon V: Deep brain stimulation: postoperative issues. Mov Disord 2006; 21(suppl 14):S219-S237.

51 Meissner W, Leblois A, Hansel D, Bioulac B, Gross CE, Benazzouz A, Boraud T: Subthalamic high-frequency stimulation resets subthalamic firing and reduces abnormal oscillations. Brain 2005;128:2372-2382.

52 Benazzouz A, Piallat B, Pollak P, Benabid AL: Responses of substantia nigra pars reticulata and globus pallidus complex to high-frequency stimulation of the subthalamic nucleus in rats: electrophysiological data. Neurosci Lett 1995;189:77-80.
53 Maurice N, Thierry AM, Glowinski J, Deniau JM: Spontaneous and evoked activity of substantia nigra pars reticulata neurons during high-frequency stimulation of the subthalamic nucleus. J Neurosci 2003;23:99299936.

54 Nimura T, Yamaguchi K, Ando T, Shibuya S, Oikawa T, Nakagawa A, Shirane R, Itoh M, Tominaga T: Attenuation of fluctuating striatal synaptic dopamine levels in patients with Parkinson disease in response to subthalamic nucleus stimulation: a positron emission tomography study. J Neurosurg 2005;103:968-973.

55 Shon YM, Lee KH, Goerss SJ, Kim IY, Kimble C, Van Gompel JJ, Bennet K, Blaha CD, Chang SY: High-frequency stimulation of the subthalamic nucleus evokes striatal dopamine release in a large animal model of human DBS neurosurgery. Neurosci Lett 2010;475:136-140.

-56 Foffani G, Priori A: Deep brain stimulation in Parkinson's disease can mimic the $300-\mathrm{Hz}$ subthalamic rhythm. Brain 2006;129:e59e60.

$\checkmark 57$ Foffani G, Priori A, Egidi M, Rampini P, Tamma F, Caputo E, Moxon KA, Cerutti S, Barbieri S: $300-\mathrm{Hz}$ subthalamic oscillations in Parkinson's disease. Brain 2003;126:21532163.

-58 Hilker R, Voges J, Thiel A, Ghaemi M, Herholz K, Sturm V, Heiss WD: Deep brain stimulation of the subthalamic nucleus versus levodopa challenge in Parkinson's disease: measuring the on- and off-conditions with FDG-PET. J Neural Transm 2002;109:12571264.

59 Baudrexel S, Witte T, Seifried C, von Wegner F, Beissner F, Klein J, Steinmetz H, Deichmann R, Roeper J, Hilker R: Resting state FMRI reveals increased subthalamic nucleus-motor cortex connectivity in Parkinson's disease. Neuroimage 2011;55:1728-1738.

60 Dorval AD, Russo GS, Hashimoto T, Xu W Grill WM, Vitek JL: Deep brain stimulation reduces neuronal entropy in the MPTP-primate model of Parkinson's disease. J Neurophysiol 2008;100:2807-2818.

61 Hammond C, Ammari R, Bioulac B, Garcia $\mathrm{L}$ : Latest view on the mechanism of action of deep brain stimulation. Mov Disord 2008;23: 2111-2121.

62 Montgomery EB Jr, Gale JT: Mechanisms of action of deep brain stimulation. Neurosci Biobehav Rev 2008;32:388-407. 